\title{
Reliability-Based Multidisciplinary Design Optimization under Correlated Uncertainties
}

\author{
Huanwei Xu, Xin Wang, Wei Li, Mufeng Li, Suichuan Zhang, and Cong Hu \\ School of Mechatronics Engineering, University of Electronic Science and Technology of China, Chengdu, \\ Sichuan 611731, China \\ Correspondence should be addressed to Huanwei Xu; xhw2211@163.com
}

Received 14 June 2017; Revised 30 July 2017; Accepted 17 September 2017; Published 18 October 2017

Academic Editor: Matteo Bruggi

Copyright (C) 2017 Huanwei Xu et al. This is an open access article distributed under the Creative Commons Attribution License, which permits unrestricted use, distribution, and reproduction in any medium, provided the original work is properly cited.

\begin{abstract}
Complex mechanical system is usually composed of several subsystems, which are often coupled with each other. Reliabilitybased multidisciplinary design optimization (RBMDO) is an efficient method to design such complex system under uncertainties. However, the present RBMDO methods ignored the correlations between uncertainties. In this paper, through combining the ellipsoidal set theory and first-order reliability method (FORM) for multidisciplinary design optimization (MDO), characteristics of correlated uncertainties are investigated. Furthermore, to improve computational efficiency, the sequential optimization and reliability assessment (SORA) strategy is utilized to obtain the optimization result. Both a mathematical example and a case study of an engineering system are provided to illustrate the feasibility and validity of the proposed method.
\end{abstract}

\section{Introduction}

The conventional MDO is a deterministic method, which considers the loads, material properties, geometry dimensions, and so on as deterministic factors. However, uncertainties often exist during entire life cycle of the mechanical systems. The MDO results might be inaccurate due to these uncertainties and their propagations between subsystems. For instance, Du and Chen [1] proposed an integrated MDO method by using the system uncertainty analysis (SUA) and concurrent subsystems uncertainty analysis (CSSUA) methods [2]. Gu et al. [3,4] proposed an implicit uncertainty delivery method to estimate the uncertainties. Sankararaman et al. $[5,6]$ utilized Bayesian statistics to solve the presence of incomplete information in actual design situations. Yao et al. [7] presented a RBMDO procedure based on combined probability and evidence theory to solve the problem under aleatory and epistemic uncertainties. Jiang et al. [8] proposed a spatial-random-process (SRP) based on multidisciplinary uncertainty analysis (MUA) method to address both aleatory and epistemic uncertainties. For more details on how to conduct mechanical design under uncertainties, including physics-based and reliability-based design, one can refer to [9-18].
Recently, reliability analysis in MDO has been paid more attention. Agarwal et al. [15] quantified uncertainties using evidence theory and then applied the sequential approximate optimization approach to drive the design optimization. $\mathrm{Du}$ and Chen [16] presented a modified concurrent subsystem uncertainty analysis (MCSSUA) method for uncertainty analysis in MDO. Park et al. $[17,18]$ combined reliability-based design optimization, possibility-based design optimization, and robust design optimization methods with the MDO method to obtain the reliable results. Lin and Gea [19] developed a probabilistic gradient-based transformation method (PGTM) to solve the complex design optimization problems under design uncertainties. Ahn and Kwon [20] presented an efficient reliability-based multidisciplinary design optimization strategy using BLISS. For ensuring the operational reliability of engineering critical components, Zhu et al. [2123] studied a unified failure criterion for structural integrity analysis and life assessment of critical components operating under harsh conditions.

The above-mentioned reliability-based multidisciplinary design optimization (RBMDO) methods intend to quantify and model various uncertainties with an assumption that these uncertainties are independent of each other. However, uncertainties are often correlated in real mechanical design 
problems [24-26]. According to this, a new MDO approach based on the ellipsoidal set theory and first-order reliability method is proposed to handle correlated uncertainties in this paper.

The remainder of the paper is organized as follows: Section 2 investigates the characteristics of correlated uncertainties. In Section 3, a mathematical model based on the ellipsoidal set theory and first-order reliability method is elaborated to estimate the correlated uncertainties. Section 4 presents a new RBMDO method considering correlated uncertainties. In Section 5, the proposed method is illustrated with a mathematical example and an engineering example. Conclusions are made in Section 6.

\section{Correlated Uncertainty Analysis}

2.1. Definition of Correlated Uncertainties. Let $X$ present $n$ dimensional uncertainties, $X \in R^{n}$, and all possible values of uncertainties can be described by a multidimensional ellipsoidal set $E$ :

$$
X \in E=\left\{X \mid(X-\bar{X})^{T} Q(X-\bar{X}) \leq \varepsilon^{2}\right\},
$$

where $\bar{X}$ is the nominal value of design variable, $Q$ is the symmetric characteristic matrix which determines the shape and principal axis direction of the ellipsoid, and $\varepsilon$ is the radius of the ellipsoid.

Let $X^{U}, X^{L}$ be the low and upper bounds of $X$, respectively, and the nominal value $\bar{X}$ can be obtained by

$$
\bar{X}=\frac{X^{L}+X^{U}}{2} \text {. }
$$

The ellipsoidal model under correlated uncertainties is derived as follows:

$$
\begin{aligned}
G= & (X-\bar{X})^{T} C^{-1}(X-\bar{X})=\left(\begin{array}{ccc}
x_{1} & -\bar{x}_{1} \\
\vdots & \vdots \\
x_{n}-\bar{x}_{n}
\end{array}\right)^{T} \\
& \cdot\left(\begin{array}{cccc}
D\left(x_{1}\right) & \operatorname{Cov}\left(x_{1}, x_{2}\right) & \cdots & \operatorname{Cov}\left(x_{1}, x_{n}\right) \\
\operatorname{Cov}\left(x_{2}, x_{1}\right) & D\left(x_{2}\right) & \cdots & \operatorname{Cov}\left(x_{2}, x_{n}\right) \\
\vdots & \vdots & \ddots & \vdots \\
\operatorname{Cov}\left(x_{n}, x_{1}\right) & \operatorname{Cov}\left(x_{n}, x_{2}\right) & \cdots & D\left(x_{n}\right)
\end{array}\right) \\
& \left(\begin{array}{c}
x_{1}-\bar{x}_{1} \\
\vdots \\
\vdots \\
x_{n}-\bar{x}_{n}
\end{array}\right) \leq \varepsilon^{2},
\end{aligned}
$$

where $\operatorname{Cov}\left(x_{i}, x_{j}\right)$ is the covariance of $x_{i}$ and $x_{j} \cdot \rho_{x_{i} x_{j}}$ is the correlation coefficient of $x_{i}$ and $x_{j}$; the correlation between uncertainties $x_{i}$ and $x_{j}$ can be expressed as

$$
\operatorname{Cov}\left(x_{i}, x_{j}\right)=\rho_{x_{i} x_{j}} \sigma_{x_{i}} \sigma_{x_{j}}=\rho_{x_{i} x_{j}} \sqrt{D\left(x_{i}\right)} \sqrt{D\left(x_{j}\right)},
$$

where $\sigma_{x_{i}}$ is the standard deviation of $x_{i}$ and $D\left(x_{i}\right)$ is the variance of $x_{i}$.

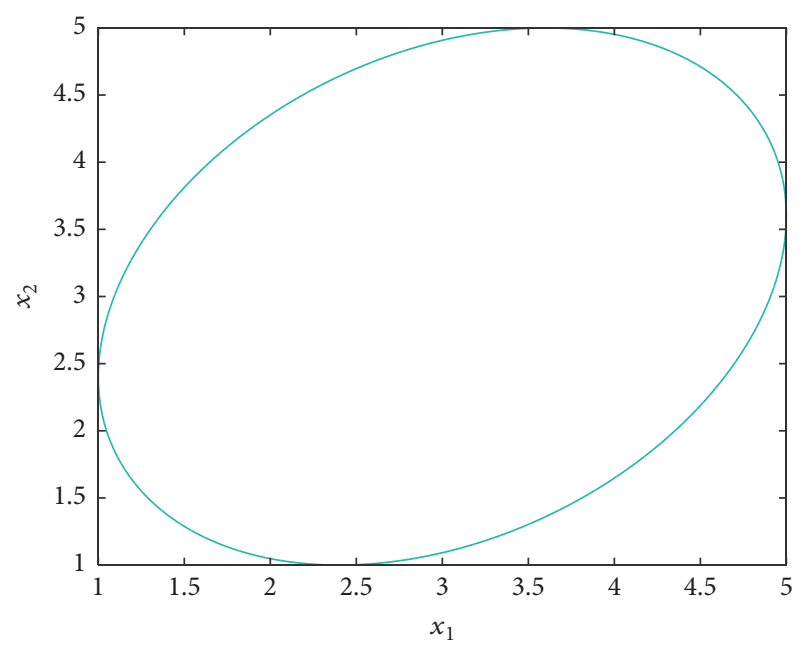

FIGURE 1: Schematic diagram of two correlated uncertainties.

In this analysis, take two correlated uncertainties as an example: $X=\left[x_{1}, x_{2}\right]$, and $x_{1} \in[1,5], x_{2} \in[1,5]$. The correlation coefficient matrix $\rho_{0}$ is as follows:

$$
\rho_{0}=\left(\begin{array}{cc}
1 & 0.3 \\
0.3 & 1
\end{array}\right)
$$

and the covariance matrix $C_{0}$ is

$$
C_{0}=\left(\begin{array}{cc}
\frac{4}{3} & 0.4 \\
0.4 & \frac{4}{3}
\end{array}\right)
$$

Using (3), the corresponding ellipsoidal model $G_{0}$ is constructed as follows:

$$
\begin{aligned}
G_{0} & =(x-\bar{x})^{T} C_{0}(x-\bar{x}) \\
& =\left(\begin{array}{l}
x-3 \\
x-3
\end{array}\right)^{T}\left(\begin{array}{cc}
\frac{4}{3} & 0.4 \\
0.4 & \frac{4}{3}
\end{array}\right)^{-1}\left(\begin{array}{l}
x-3 \\
x-3
\end{array}\right) \leq \varepsilon^{2} .
\end{aligned}
$$

According to the minimal volume method [27], we have $\varepsilon=3$. The corresponding schematic diagram of these two correlated uncertainties is shown in Figure 1.

\section{Reliability Analysis under Correlated Uncertainties}

In most engineering optimization problems, designers assume that uncertainties are independent of each other. However, correlations often exist among multisource uncertainties. The conventional reliability methods, such as first and second reliability methods, cannot be used to solve such problems directly.

3.1. Reliability-Based Design Optimization. Reliability-based design optimization is a commonly used method to obtain 
design optimization results with acceptable lower failure probability. The reliability design optimization model can be expressed as follows:

$$
\begin{aligned}
\min & f(X, P) \\
\text { s.t. } & P\left[g_{i}(X, P) \leq 0\right] \geq\left[R_{i}\right],
\end{aligned}
$$

where $f$ is the objective function, $X$ is a vector of random design variables, $P$ is a vector of design parameters, $g_{i}(X, P) \leq$ 0 is the $i$ th constraint, which is also called the limit state function or performance function in reliability analysis, $\left[R_{i}\right]$ represents the minimum reliability accepted by designers, and $P[g(X, P) \leq 0]$ denotes the probability of design variable $X$ in the feasible region.

First-order reliability method (FORM) is a traditional method to calculate the reliability. FORM simplifies the integral operation and approximates limit state function by using the mean and variance of random variables $[28,29]$. FORM requires that random variables are independent standard normal distributions. However, the uncertainties are often correlated in real engineering issues. Accordingly, this situation needs to transform correlated uncertainties into independent uncertainties during reliability analysis [30, 31].

For independent uncertainties $Y$, we need to transform it into a standard normal distribution $U$ by

$$
U=\frac{Y-\mu_{Y}}{\sigma_{Y}}
$$

Then, the Taylor approximation with respect to limit state function can be derived. In order to improve calculation accuracy of Taylor approximation, the value of the probability density at the expansion point $\left(U^{*}\right)$ should be as follows: the bigger the better. When leaving this point the value of probability density function will decline at a faster rate. Thus, $U^{*}$ is also called the most probable point (MPP). The mathematical model for solving MPP is shown as follows:

$$
\begin{aligned}
& \min \beta=\|u\|=\sqrt{u^{T} u}=\sqrt{\sum_{i=1}^{n}\left(\frac{Y_{i}-u_{Y_{i}}}{\sigma_{Y_{i}}}\right)^{2}} \\
& \text { s.t. } g(U)=0,
\end{aligned}
$$

where $\|U\|=\sqrt{u_{1}^{2}+u_{2}^{2}+\cdots+u_{n}^{2}}$; in geometric space MPP is the closest point on the limit state function to the origin of coordinates. Let $\beta=\left\|U^{*}\right\|$, which is the reliability index.

Inverse reliability strategy is introduced to solve reliability optimization problems [32]. The strategy tries to find percentage performance $g^{p}$ in the case of a given probability $p$ :

$$
P\left[g(Y, P) \leq g^{p}\right]=p .
$$

Then, this leads to a new performance function:

$$
g^{\prime}(Y, P)=g(X, P)-g^{p} .
$$

The MPP should satisfy the following formula:

$$
\beta=\left|\Phi^{-1}(p)\right|
$$

The reliability problem can be transformed to find the MPP of the maximum performance function on the circle $\beta$, and the following optimization model can be established:

$$
\begin{aligned}
\max & g\left(U_{Y}, P\right) \\
\text { s.t. } & \left\|u_{Y}\right\|=\beta .
\end{aligned}
$$

3.2. Transformation of Correlated Uncertainties. As mentioned before, current reliability methods cannot be applied to handle correlated uncertainties directly. When uncertainties are statistically correlated, Rosenblatt transformation, Nataf transformation, and orthogonal transformation are often used to handle these uncertainties in reliability analysis. Among them, Rosenblatt transformation needs to know the joint cumulative distribution function of variables in advance. Nataf transformation takes into account the change of correlation caused by transformation process. Orthogonal transformation is relatively simple and easy to be implemented, but it has a premise that the transformation process is basically not to change the relevance of the variables. More details on these three methods can be found in [30]. Since orthogonal transform has shown higher computation efficiency, in this paper, it is used to deal with the correlated uncertainties.

Orthogonal transformation can be applied to handle correlated random variables. Let vector $X$ present the normal correlated random uncertainties: $X=\left(x_{1}, x_{2}, \ldots, x_{n}\right)^{T}$. The covariance $C$ of $X$ can be expressed as follows:

C

$$
=\left(\begin{array}{cccc}
D\left(x_{1}\right) & \operatorname{Cov}\left(x_{1}, x_{2}\right) & \cdots & \operatorname{Cov}\left(x_{1}, x_{n}\right) \\
\operatorname{Cov}\left(x_{2}, x_{1}\right) & D\left(x_{2}\right) & \cdots & \operatorname{Cov}\left(x_{2}, x_{n}\right) \\
\vdots & \vdots & \ddots & \vdots \\
\operatorname{Cov}\left(x_{n}, x_{1}\right) & \operatorname{Cov}\left(x_{n}, x_{2}\right) & \cdots & D\left(x_{n}\right)
\end{array}\right)
$$

Let the matrix $A$ consist of regularized eigenvectors of $C$. The matrix $A$ should satisfy the condition that $C$ can be transformed to a diagonal matrix through matrix operation $A^{T} C A$. Let $X=A Y$, since $A^{-1}=A T$; this leads to

$$
Y=A^{-1} X=A^{T} X,
$$

where $Y$ is the linear independent vector and the covariance of $Y$ is $D_{Y}=\operatorname{diag}\left[\sigma_{Y_{i}}^{2}\right]_{n \times n}$. The mean value and covariance can be expressed by the following formulas, respectively:

$$
\begin{gathered}
u_{Y}=A^{T} E_{X} \\
D_{Y}=A^{T} C A .
\end{gathered}
$$

Linear combination of normal random variables is still a normal random variable. Since the uncorrelated normal random variables are equivalent to the independent normal random variables, $Y$ is the independent normal random variable. Then, the performance function is

$$
Z=g(X)=g_{X}(A Y)=g_{Y}(Y) .
$$

According to (16), the reliability can be calculated by utilizing FORM. 


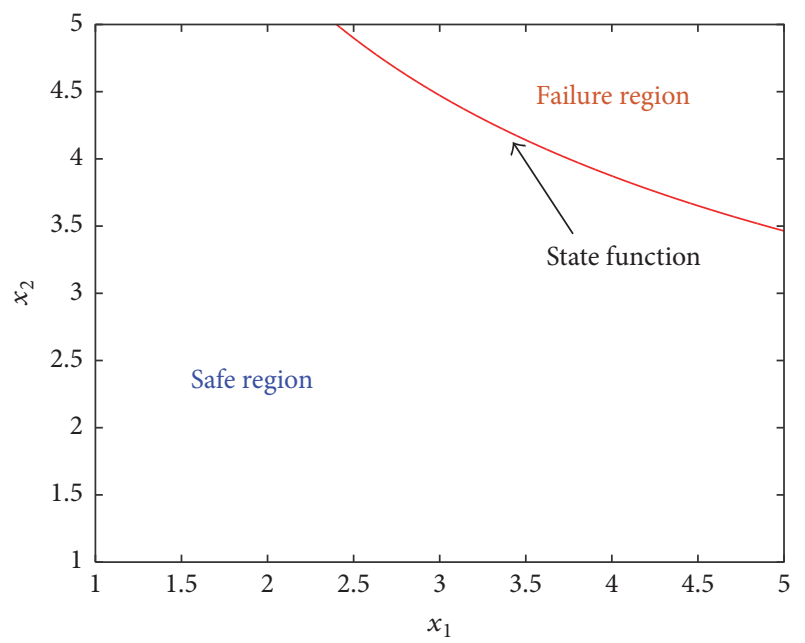

FIGURE 2: Safe region and failure region of limit state function (without considering correlations).

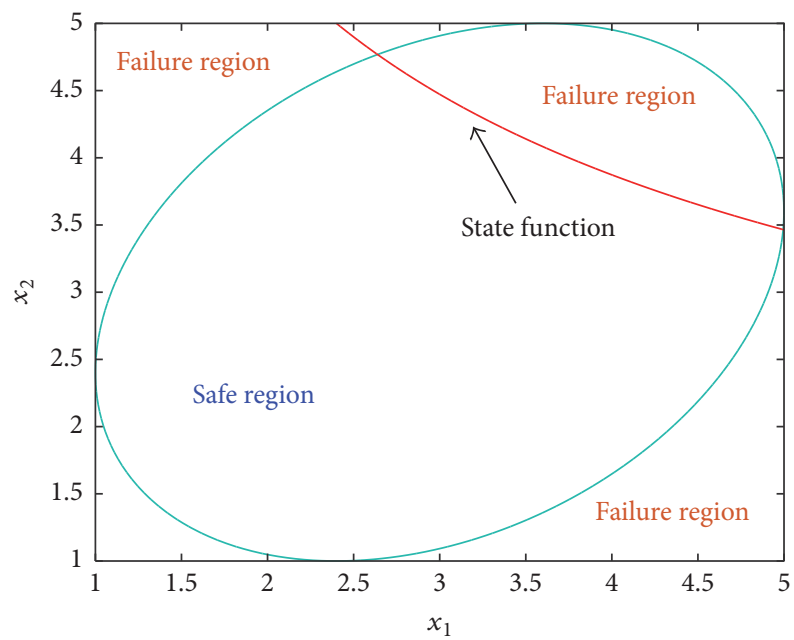

Figure 3: Safe region and failure region of limit state function (considering correlations).

3.3. The Effect of Correlated Uncertainties on Reliability. The correlated uncertainties generally exist in real mechanical design problems. Not considering the correlations between uncertainties might lead to inaccurate design solutions. In this section, a simple mathematical model is taken as an example to illustrate the effect of correlated uncertainties on reliability. Let $x_{1}$ and $x_{2}$ be design variables, and $x_{1} \in$ $\left[x_{1}{ }^{l}, x_{1}{ }^{u}\right], x_{2} \in\left[x_{2}{ }^{l}, x_{2}{ }^{u}\right]$. The limit state function is given as follows:

$$
g_{X}=x_{1} x_{2}^{2}-60 \leq 0 .
$$

If the correlation between the two uncertainties is not considered, the safe region and failure region of limit state function can be obtained as shown in Figure 2 .

Assuming that these two uncertainties are correlated and the correlation coefficient $\rho_{x_{1} x_{2}}$ is 0.3 , the corresponding safe region and failure region are shown in Figure 3.

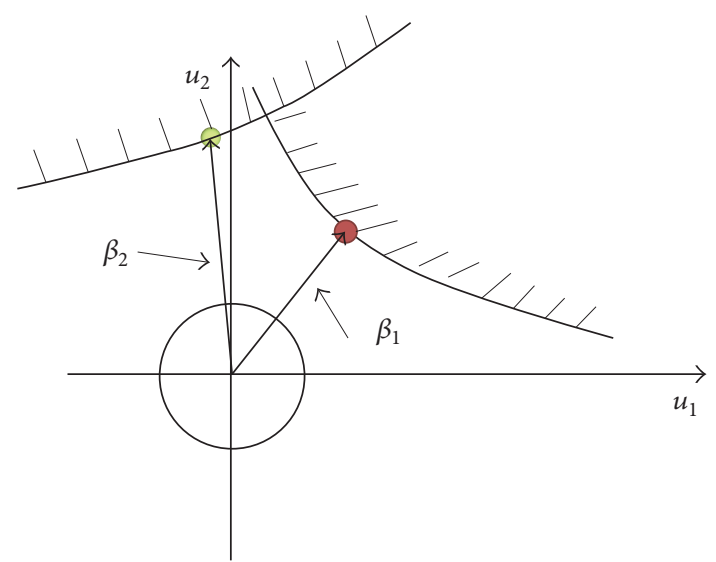

FIGURE 4: Comparison of reliability results.

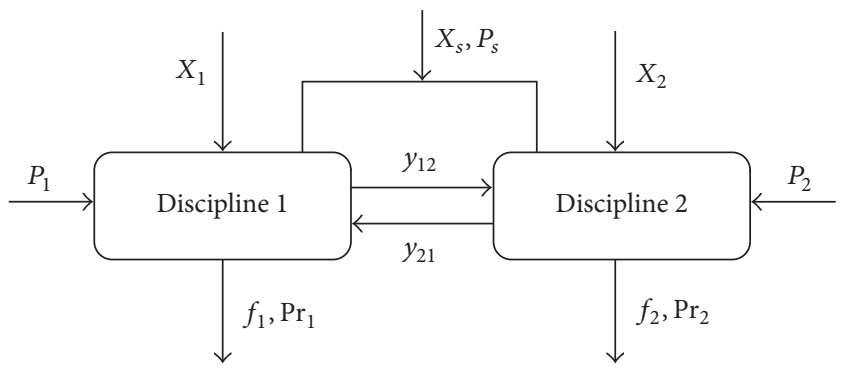

FIgURE 5: Scheme of two subsystems.

Let $x_{1}$ and $x_{2}$ be normal distributions with mean 3 and variance 0.3 ; the correlation coefficient $\rho_{x_{1} x_{2}}$ is 0.3 . Firstly, the orthogonal transformation method is utilized to transform correlated $X$ to independent $Y$; then $Y$ is transformed to the standard normal space $U$ for reliability analysis by using FORM. Figure 4 shows that when the correlations between uncertainties are not considered, the corresponding reliability index is $\beta_{1}=2.26$ and when the correlations of uncertainties are considered, the corresponding reliability index is $\beta_{2}=3.27$. From this simple mathematical example, a conclusion can be drawn that correlations of uncertainties have shown an influence on reliability.

\section{Reliability-Based MDO under Correlated Uncertainties}

Since a complex designed mechanical system often involves many subsystems, in this section, reliability-based multidisciplinary design optimization is carried out by considering correlated uncertainties.

4.1. RBMDO. In this study, we assume that a system is composed of two subsystems, which is shown in Figure 5.

According to Figure 5, the model of RBMDO is as follows:

$$
\begin{array}{ll}
\min & f_{i}\left(X_{s}, P_{s}, X_{i}, P_{i}, y_{i j}\right) \\
\text { s.t. } & \operatorname{Pr}\left[g_{i}\left(X_{s}, P_{s}, X_{i}, P_{i}, y_{i j}\right) \leq 0\right] \geq[R]
\end{array}
$$




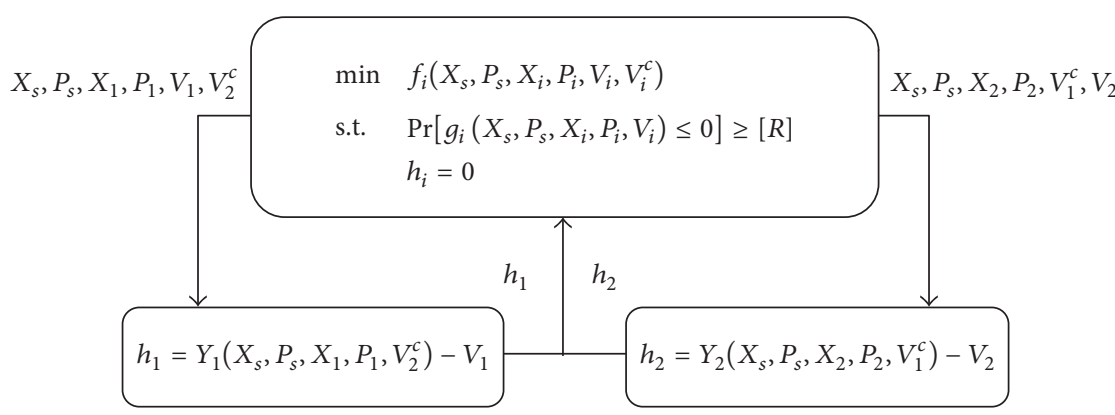

FIGURE 6: Framework of SAND-RBMDO.

$$
\begin{aligned}
& h_{i}\left(X_{s}, P_{s}, X_{i}, P_{i}, y_{i j}\right)=0 \\
& \quad \text { where } y_{i j}=Y_{i}\left(X_{s}, P_{s}, X_{i}, P_{i}, y_{j i}\right), i=1,2,
\end{aligned}
$$

where $X_{s}, P_{s}$ are the share variables and share parameters, respectively, $y_{i j}$ is the coupled state variable from $i$ th subsystem to $j$ th subsystem, $X_{i}$ and $P_{i}$ are the local variables and local parameters of $i$ th subsystem, respectively, $g_{i}$ and $f_{i}$ represent the constraints and objective function of $i$ th subsystem, respectively, $h_{i}$ is the equality constraint, $Y_{i}$ is the function to obtain coupled state variable $y_{i j}$, and $[R]$ is the designed reliability.

4.2. SAND-RBMDO. Simultaneous analysis and design (SAND) is a typical MDO method. It utilizes optimization solver to substitute the system and/or subsystem analysis, which reduces the high analysis cost. In SAND method, in order to reconcile the inconsistency caused by coupling characteristics of multidiscipline system, the coupled variables are treated as design variables. The framework of SAND-RBMDO is elaborated as shown in Figure 6.

In Figure $6, V_{i}$ are state variables and $V_{i}^{c}$ are complement variables. The deterministic MDO optimization for the $k$ th iteration can be expressed as

$$
\begin{array}{ll}
\min & f_{i}\left(X_{s}^{*, k-1}, P_{s}, X_{i}^{*, k-1}, P_{i}, V_{i}, V_{i}^{c}\right) \\
\text { s.t. } & g_{i}\left(\bar{X}_{s}, P_{s}, \bar{X}_{i}, P_{i}, V_{i}, V_{i}^{c}\right) \leq 0 \\
& h_{i}\left(\bar{X}_{s}, P_{s}, \bar{X}_{i}, P_{i}, V_{i}, V_{i}^{c}\right)=0
\end{array}
$$

$$
i=1,2, \ldots, n \text {, }
$$

where $\bar{X}_{s}, \bar{X}_{i}$ are the nominal values, $X_{i}^{*, k-1}, X_{i}^{*, k-1}$ are the MPP point obtained from the $(k-1)$ th iteration of reliability analysis, and $P_{s}, P_{i}, V_{i}, V_{c}$ are optimization results obtained from deterministic optimization process. The corresponding inverse reliability optimization formula is

$$
\begin{array}{ll}
\max & g_{i}\left(P_{s}, P_{i}, U_{X_{s}}, U_{X_{i}}, V_{i}, V_{i}^{c}\right) \\
\text { s.t. } & \left\|\left(U_{X_{s}}, U_{X_{i}}\right)\right\|=\beta,
\end{array}
$$

where $\beta$ is the reliability index.
4.3. CC-SORA-RBMDO. The reliability-based MDO under correlated uncertainties is investigated in this section. The correlations between uncertainties are described by ellipsoidal model in the deterministic MDO process. When the reliability analysis is implemented, the CC-FORM method is used to calculate the reliability index. In order to improve computation efficiency, SORA strategy is applied $[33,34]$. The procedure of CC-SORA-RBMDO is shown in Figure 7.

According to Figure 7, the optimization steps of CCSORA-RBMDO are given as follows.

Step 1. According to the correlation coefficient of any two uncertainties, obtain the covariance matrix of correlated uncertainties (see (4)); then construct ellipsoidal model (see (3)). The ellipsoidal model is added as constraint $G_{i}\left(P_{s}, P_{i}\right) \leq$ $\varepsilon^{2}$ in the MDO model. The deterministic MDO (see (22)) is implemented.

Step 2. The optimization result of deterministic MDO can be obtained. The corresponding inverse reliability analysis (see (23)) is implemented by utilizing the FORM method.

Step 3. If the optimization convergence and the reliability requirements of constraints are satisfied, then end the optimization process. Otherwise implement the next step.

Step 4. According to the values of MPP, generate new mean values of correlated uncertainties and implement new deterministic MDO.

A corresponding flowchart of the proposed procedure is given in Figure 8.

\section{Case Studies}

In this section, both a mathematical example and a case study of an engineering system are utilized to illustrate feasibility and validity of the proposed method.

5.1. A Mathematical Example. A classical MDO problem is introduced to illustrate the proposed MDO method [35]. The problem has two subsystems. Coupling relationship exists between these two subsystems, as shown in Figure 9. The mathematical expression is listed as follows:

$$
\begin{array}{ll}
\min & f=\left(p_{s}+x_{s}\right)^{2}+\left(p_{1}+x_{1}\right)^{2}+\left(p_{2}+x_{2}\right)^{2} \\
\text { s.t. } & \operatorname{Pr}\left[g_{1}=x_{1} x_{2}-\left(p_{s}+x_{s}+2 p_{1}+2 y_{21}\right) \leq 0\right]
\end{array}
$$




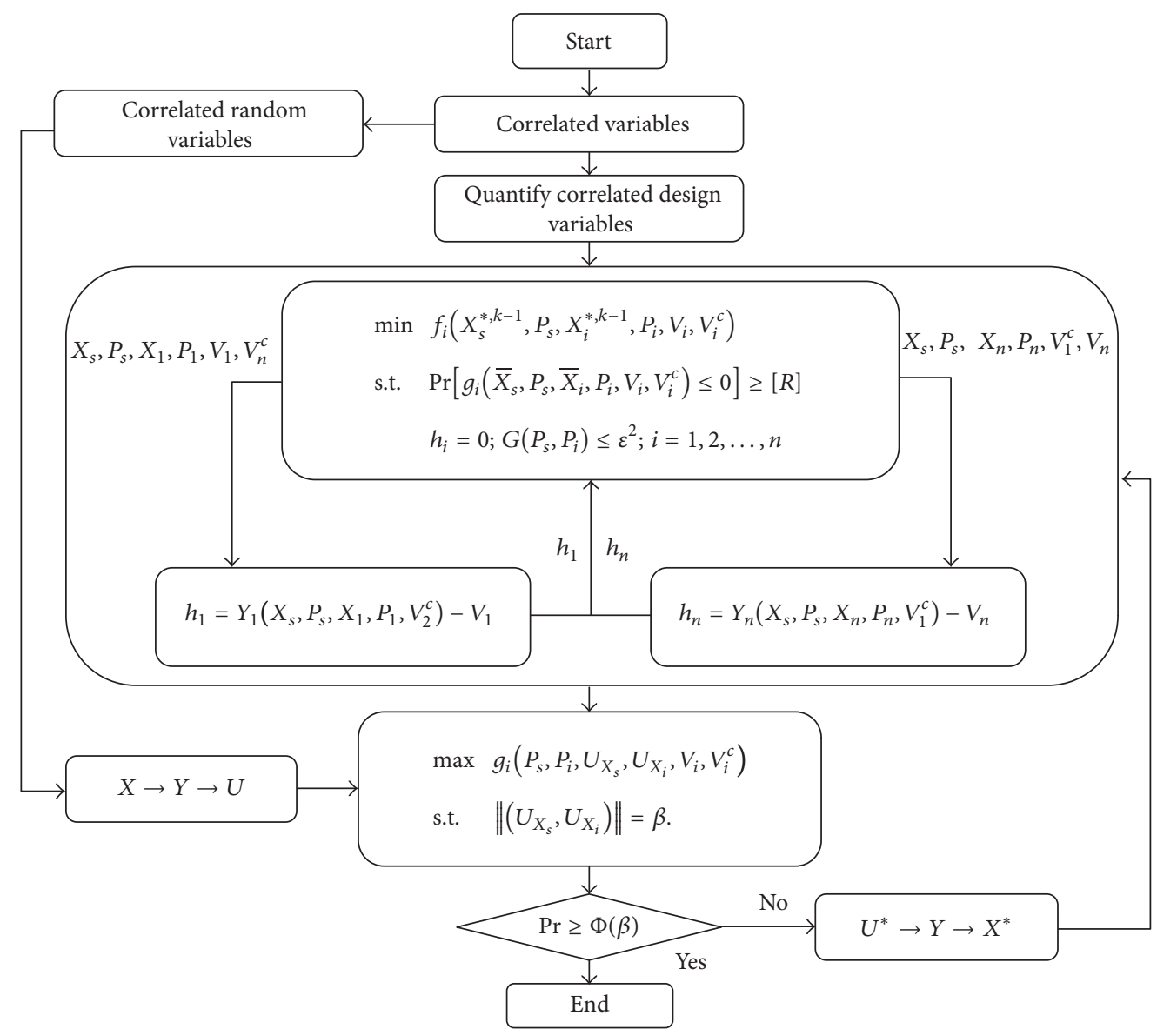

FIgURE 7: The procedure of CC-SORA-RBMDO.

TABLE 1: The information of design variables and design parameters.

\begin{tabular}{lccccc}
\hline$x_{s}$ & $x_{1}$ & $x_{2}$ & $p_{1}$ & $p_{2}$ & $p_{3}$ \\
\hline$[0,5]$ & {$[0,5]$} & {$[0,5]$} & 0 & 5 & 1 \\
\hline
\end{tabular}

$$
\begin{aligned}
& \geq\left[R_{1}\right] \\
& \operatorname{Pr}\left[g_{2}=5 p_{s}+5 x_{s}+3 p_{2}-4 y_{12}-x_{2}-3 \leq 0\right] \\
& \geq\left[R_{2}\right] \\
& y_{12}=p_{s}+x_{s}+p_{1}+y_{21} \\
& y_{21}=p_{s}+x_{s}+p_{2}-y_{12},
\end{aligned}
$$

where $f$ is the objective function, $x_{s}, p_{s}$ are the sharing design variable and sharing random design parameter, respectively, $x_{1}, p_{1}$ are the local design variable and local random design parameter of subsystem $1, x_{2}, p_{2}$ are the local design variable and local random design parameter of subsystem 2 , and $y_{12}$ and $y_{21}$ are the coupled state variables.

The information of design variables $x_{s}, x_{1}, x_{2}$ and design parameters $p_{1}, p_{2}, p_{3}$ is listed in Table 1 .
The mean values of $x_{s}, x_{1}, x_{2}$ are $2.5,2.5,2.5$. The correlation coefficient matrix $\rho_{X}$ of design variables is

$$
\rho_{X}=\left(\begin{array}{ccc}
1 & 0.2 & 0.1 \\
0.2 & 1 & -0.3 \\
0.1 & -0.3 & 1
\end{array}\right)
$$

The covariance matrix $C_{X}$ is

$$
C_{X}=\left(\begin{array}{rrr}
\frac{25}{12} & \frac{5}{12} & \frac{5}{24} \\
\frac{5}{12} & \frac{25}{12} & -\frac{5}{8} \\
\frac{5}{24} & -\frac{5}{8} & \frac{25}{12}
\end{array}\right)
$$

The corresponding ellipsoidal model $G_{X}$ is derived according to (3) as follows:

$$
G_{X}=(X-\bar{X})^{T} C^{-1}(X-\bar{X})=\left(\begin{array}{l}
x_{s}-2.5 \\
x_{1}-2.5 \\
x_{2}-2.5
\end{array}\right)^{T}
$$






FIGURE 8: Flowchart of the proposed method for CC-SORA-RBMDO.

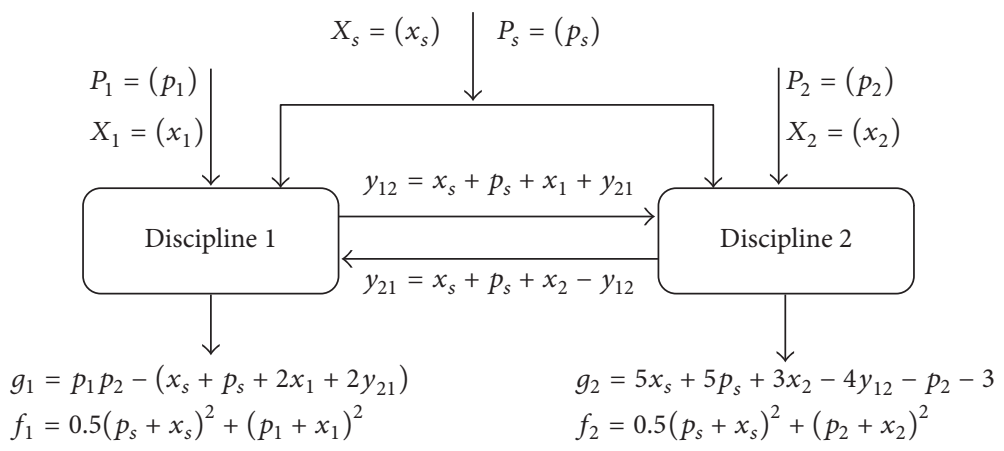

FIgURE 9: The diagram of mathematical example.

$$
\left(\begin{array}{rrr}
\frac{25}{12} & \frac{5}{12} & \frac{5}{24} \\
\frac{5}{12} & \frac{25}{12} & -\frac{5}{8} \\
\frac{5}{24} & -\frac{5}{8} & \frac{25}{12}
\end{array}\right)^{-1}\left(\begin{array}{l}
x_{s}-2.5 \\
x_{1}-2.5 \\
x_{2}-2.5
\end{array}\right) \leq \varepsilon^{2}
$$

For random design parameters, Rosenblatt transformation, orthogonal transformation, or Nataf transformation is used to convert correlated design parameters to independent design parameters. The correlation coefficient matrix $\rho_{P}$ of design parameters is

$$
\rho_{P}=\left(\begin{array}{ccc}
1 & 0.4 & 0.3 \\
0.4 & 1 & 0.3 \\
0.3 & 0.3 & 1
\end{array}\right)
$$

The covariance matrix $C_{P}$ is

$$
C_{P}=\left(\begin{array}{ccc}
0.1 & 0.0894 & 0.03 \\
0.0894 & 0.5 & 0.0671 \\
0.03 & 0.0671 & 0.1
\end{array}\right) .
$$

Normalize the eigenvectors of $C_{P}$ :

$$
A=\left(\begin{array}{ccc}
-0.7871 & -0.5795 & 0.2114 \\
0.0679 & 0.2592 & 0.9634 \\
0.6131 & -0.7723 & 0.1646
\end{array}\right) .
$$

According to the following transformation

$$
P=A Y,
$$

we have

$$
\begin{aligned}
& p_{1}=-0.7871 y_{1}-0.5795 y_{2}+0.2114 y_{3} \\
& p_{2}=0.0679 y_{1}+0.2592 y_{2}+0.9634 y_{3} \\
& p_{3}=0.6131 y_{1}-0.7723 y_{2}+0.1646 y_{3} .
\end{aligned}
$$


TABLE 2: Optimization results.

\begin{tabular}{lccccrr}
\hline & $x_{s}$ & $x_{1}$ & $x_{2}$ & $f$ & $\operatorname{Pr}_{1}$ & $\operatorname{Pr}_{2}$ \\
\hline CASE 1 & 3.1066 & 1.1928 & 2.2321 & 58.4484 & 0.9800 & 0.9801 \\
CASE 2 & 3.0474 & 1.2332 & 2.2410 & 58.6440 & 0.9800 & 0.9893 \\
CASE 3 & 3.1742 & 0.5549 & 2.0549 & 50.2650 & 0.9901 & 0.9886 \\
CASE 4 & 3.2704 & 0.6254 & 2.0549 & 52.1088 & 0.9893 & 0.9893 \\
\hline
\end{tabular}

Through converting the vector $P$ into the linear independent vector $Y$, the covariance matrix of $Y$ is $D_{Y}=$ $\operatorname{diag}\left[\sigma_{Y_{i}}^{2}\right]_{n \times n}$. The mean value and variance of $Y$ can be expressed as $u_{Y}=A^{T} E_{P}$ and $D_{Y}=A^{T} C_{P} A$. The corresponding function $g(P)=g_{P}(A Y)=g_{Y}(Y)$; then we can convert $Y$ to the standard normal space by

$$
U=\frac{Y-E_{Y}}{\sqrt{D_{Y}}} .
$$

Then, the FORM method is used to obtain MPP $\left(U^{*}\right)$. When the deterministic MDO is implemented, the vector $P$ can be obtained by (31)-(33).

The deterministic optimization problem can be solved by using SAND method. The MPP $\left(u_{s}^{*, c,(1)}, u_{1}^{*, c,(1)}, u_{2}^{*, c,(1)}\right)$, $\left(u_{s}^{*, c,(2)}, u_{1}^{*, c,(2)}, u_{2}^{*, c,(2)}\right)$ of $g_{1}$ and $g_{2}$ can be obtained from the previous cycle of reliability analysis. Then deterministic MDO optimization can be implemented by switching random variables from the $U$ space into the $P$ space. The frame of deterministic MDO is as follows:

$$
\begin{array}{ll}
\min & f=\left(x_{s}+\bar{p}_{s}\right)^{2}+\left(x_{1}+\bar{p}_{1}\right)^{2}+\left(x_{2}+\bar{p}_{2}\right)^{2} \\
\text { s.t. } & g_{1}=p_{1}^{*,(1)} p_{2}^{*,(1)}-\left(x_{s}+p_{s}^{*,(1)}+2 x_{1}+2 y_{21}\right) \\
& \leq 0 \\
& g_{2}=5 x_{s}+5 p_{s}^{*,(2)}+3 x_{2}-4 y_{12}-p_{2}^{*,(2)}-3 \\
& \leq 0 \\
& y_{12}=x_{s}+p_{s}^{*,(1,2)}+x_{1}+y_{21} \\
& y_{21}=x_{s}+p_{s}^{*,(1,2)}+x_{2}-y_{12} \\
& G_{X} \leq \varepsilon^{2} \\
& P=\left[p_{s}, p_{1}, p_{2}\right] \\
& X=\left[x_{s}, x_{1}, x_{2}\right] .
\end{array}
$$

The design variables $\mathrm{DV}=\left(x_{s}, x_{1}, x_{2}, y_{12}, y_{21}\right)$; through substituting the optimization result into reliability analysis, the corresponding MPP of $g_{1}$ can be obtained by

$\max g_{1}$

$$
=\left(u_{1}+u_{1}^{c,(1)} \sigma_{1}\right)\left(u_{2}+u_{2}^{c,(1)} \sigma_{2}\right)
$$

$$
\begin{array}{r}
-\left(x_{s}+\left(u_{s}+u_{s}^{c,(1)} \sigma_{s}\right)+2 x_{1}+2 y_{21}\right) \\
\text { s.t. } \sqrt{\left(u_{s}^{c,(1)}\right)^{2}+\left(u_{1}^{c,(1)}\right)^{2}+\left(u_{2}^{c,(1)}\right)^{2}}=\beta,
\end{array}
$$

where the design variables DV $=\left(u_{s}^{c,(1)}, u_{1}^{c,(1)}, u_{2}^{c,(1)}\right)$ by considering the correlations of random variables. Similarly, the MPP of $g_{2}$ can be obtained. $R 1=R 2=\Phi(\beta)=0.98$.

The optimization results are shown in Table 2. CASE 1 is the RBMDO optimization result without considering correlations between uncertainties; CASE 2 is the RBMDO optimization result by considering correlations between design variables; CASE 3 is the RBMDO optimization result by considering correlations between design parameters; CASE 4 is the RBMDO optimization result by considering correlations between both design variables and design parameters. For this mathematical example, note from Table 2 that the optimization result of CASE 2 is similar to that of CASE 1 , which means the correlations between design variables have less effect on optimization result. The optimization results of CASE 3 and CASE 4 are different from that of CASE 1, which means the correlations between design parameters have great effect on optimization result. Apparently, correlations between uncertainties have shown significant influence on the optimization results. Therefore, for accurate and informative optimization results in practical engineering, correlations must be considered during the MDO procedure.

\subsection{An Engineering Example}

5.2.1. Problem Description. This is a design optimization problem of the four-high rolling mill stand [36]. The simplified structure diagram of roller base is shown in Figure 10.

The height $h_{1}$ of column cross-section, the height $h_{2}$ of upper beam cross-section, the height $h_{3}$ of lower beam crosssection, and the diameter $D_{1}$ of supporting roll are treated as design variables:

$$
V=\left(h_{1}, h_{2}, h_{3}, D_{1}\right)^{T}=\left(x_{1}, x_{2}, x_{3}, x_{4}\right)^{T} .
$$

The width $b_{1}$ of column cross-section, the width $b_{2}$ of upper beam cross-section, and the width $b_{3}$ of 


(1) Upper beam
(4) Supporting roll
(2) Column
(5) Working roll
(3) Lower beam

FIGURE 10: Simplified structure diagram of four-high rolling mill stand.

lower beam cross-section are treated as design parameters:

$$
P=\left(b_{1}, b_{2}, b_{3}\right)=\left(p_{1}, p_{2}, p_{3}\right) .
$$

The objective function is to minimize the stand bounce value, which is composed of six parts:

(1) The bending deformation of lower and upper beams caused by the bending moment:

$$
\begin{aligned}
f_{1} & =1.91 \times 10^{-6} \times\left(x_{1}+0.59\right)^{3} \times\left(\frac{1}{\left(p_{2} x_{2}^{3}\right)}\right. \\
& \left.+\frac{1}{\left(p_{3} x_{3}^{3}\right)}\right) \times\{1-0.75 \\
& \left.\times\left[1+\frac{\left(x_{2}+x_{3}+4.3\right) p_{2} p_{1}^{3} p_{3} x_{3}^{3}}{\left(x_{1}+0.59\right) p_{1} x_{1}^{3}\left(p_{2} x_{2}^{3}+p_{3} x_{3}^{3}\right)}\right]^{-1}\right\}
\end{aligned}
$$

(2) The bending deformation of the upper and lower beams caused by shear stress:

$$
\begin{aligned}
f_{2}= & 3.704 \times 10^{-6} \times\left(x_{1}+0.59\right) \\
& \times\left(\frac{1}{\left(x_{2} p_{2}\right)}+\frac{1}{\left(x_{3} p_{3}\right)}\right)
\end{aligned}
$$

(3) Tensile deformation of the columns:

$$
f_{3}=5.119 \times 10^{-6} \times \frac{1}{x_{1} p_{1}}
$$

(4) The bending deformation of supporting rolls caused by the bending moment:

$$
\begin{aligned}
f_{4} & =0.9671 \times 10^{-6} \times \frac{1}{x_{4}^{4}} \times\left[8\left(p_{1}+0.656\right)^{3}\right. \\
& -0.64\left(p_{1}+0.656\right)+0.64+8\left(x_{2}+0.256\right)^{3} \\
& \left.\times\left(218.8 x_{4}^{4}-1\right)\right]
\end{aligned}
$$

(5) The bending deformation of the supporting rolls caused by shear stress:

$$
\begin{aligned}
& f_{5}(x, p)=1.533 \times 10^{-5} \times \frac{1}{x_{4}^{2}} \times\left[\left(p_{1}+0.656\right)-0.2\right. \\
& \left.+\left(p_{1}+0.256\right) \times\left(14.79 x_{4}^{2}-1\right)\right]
\end{aligned}
$$

(6) The squash deformation between working rolls and supporting rolls:

$$
f_{6}=0.263 \times 10^{-4} \ln \left[0.5904 \times 10^{5} \times\left(p_{4}+0.28\right)\right] .
$$

The main constraints are the deformation, stiffness, and stress requirements. There are twelve constraints in the model:

(1) The contact strength of roll:

$$
g_{1}=0.89 \times 10^{6} \times \sqrt{1+\frac{0.28}{x_{4}}}-1.61 \times 10^{6} \leq 0
$$

(2) The bending strength of dangerous section of supporting roll:

$g_{2}=0.1678 \times 10^{6} \times\left(p_{1}+0.256\right)-0.125 \times 10^{6} \leq 0$ 
TABLE 3: Variables limits (unit: $\mathrm{mm}$ ).

\begin{tabular}{lcccc}
\hline$x_{1}$ & $x_{2}$ & $x_{3}$ & $x_{4}$ \\
\hline$\left[\begin{array}{ll}200 & 400\end{array}\right]$ & {$\left[\begin{array}{ll}500 & 800\end{array}\right]$} & {$\left[\begin{array}{ll}250 & 650\end{array}\right]$} & {$\left[\begin{array}{ll}336 & 420\end{array}\right]$} \\
\hline
\end{tabular}

(3) The composite tensile and bending strength of column $\left(\left[\sigma_{P}\right]=0.055 \times 10^{6} \mathrm{KN} / \mathrm{m}^{2}\right)$ :
TABLE 4: Uncertainties distributions (unit: $\mathrm{mm}$ ).

\begin{tabular}{lccc}
\hline Variables & Mean & $\begin{array}{c}\text { Coefficient of } \\
\text { variation }\end{array}$ & $\begin{array}{c}\text { Type of } \\
\text { distribution }\end{array}$ \\
\hline$p_{1}$ & 300 & $10 \%$ & Normal \\
$p_{2}$ & 300 & $10 \%$ & Normal \\
$p_{3}$ & 400 & $10 \%$ & Normal \\
\hline
\end{tabular}

$$
g_{3}=\frac{500}{x_{1} p_{1}}+\frac{750\left(p_{1}+0.59\right)}{x_{1}^{2} p_{1}} \times\left[\frac{1}{1+\left(x_{2}+x_{3}+4.3\right) p_{2} x_{2}^{3} p_{3} x_{3}^{3} /\left(x_{1}+0.59\right) p_{1} x_{1}^{3}\left(p_{2} x_{2}^{3}+p_{3} x_{3}^{3}\right)}\right]-\left[\sigma_{P}\right] \leq 0
$$

(4) The bending strength of upper and lower beams:

$$
\begin{aligned}
g_{4}= & \frac{1.5 \times 10^{3}\left(x_{1}+0.59\right)}{p_{2} x_{2}^{2}} \times\left\{1-\frac{1}{2\left[1+1+\left(x_{2}+x_{3}+4.3\right) p_{2} x_{2}^{3} p_{3} x_{3}^{3} /\left(p_{1}+0.59\right) p_{2} p_{1}^{3}\left(p_{2} x_{2}^{3}+p_{3} x_{3}^{3}\right)\right]}\right\}-0.055 \\
& \times 10^{6} \leq 0 \\
g_{5}= & \frac{1.5 \times 10^{3}\left(x_{1}+0.59\right)}{p_{3} x_{3}^{2}} \times\left\{1-\frac{1}{2\left[1+1+\left(x_{2}+x_{3}+4.3\right) p_{2} x_{2}^{3} p_{3} x_{3}^{3} /\left(x_{1}+0.59\right) p_{1} x_{1}^{3}\left(p_{2} x_{2}^{3}+p_{3} x_{3}^{3}\right)\right]}\right\}-0.055 \\
& \times 10^{6} \leq 0
\end{aligned}
$$

(5) The dimension restrictions:

$$
\begin{aligned}
& g_{6}=p_{1}-x_{1} \leq 0 ; \\
& g_{7}=p_{2}-x_{2} \leq 0 ; \\
& g_{8}=p_{3}-x_{3} \leq 0 ; \\
& g_{9}=0.26-p_{1} \leq 0 ; \\
& g_{10}=x_{2}-2.5 p_{2} \leq 0 ; \\
& g_{11}=x_{3}-2.5 p_{3} \leq 0
\end{aligned}
$$

(6) The weight of four-high rolling mill stand being not more than the existing similar four-high rolling mill stand's:

$$
\begin{aligned}
g_{12}= & 15.6 \\
& \times\left[2.15 x_{1} p_{1}+\left(x_{1}+0.295\right)\left(x_{2} p_{2}+x_{3} p_{3}\right)\right] \\
& -7.484 \leq 0 .
\end{aligned}
$$

As mentioned before, the system can be divided to the three subsystems, namely, column subsystem, beam subsystem, and support roll subsystem. The frame is shown in Figure 11.

The variables limits and uncertainties distributions of four-high mill stand are shown in Tables 3 and 4, respectively.
5.2.2. Reliability-Based MDO of Four-High Rolling Mill Stand. Take $g_{3}, g_{4}, g_{5}, g_{12}$ as probabilistic reliability constraints and accordingly reliability lever is $R=R n=\Phi(\beta)=0.98$. The correlation matrix of design variables $x_{1}, x_{2}$, and $x_{3}$ is

$$
\rho=\left(\begin{array}{ccc}
1 & -0.2 & -0.3 \\
-0.2 & 1 & 0.2 \\
-0.3 & 0.2 & 1
\end{array}\right)
$$

The correlation matrix of design parameters $p_{1}, p_{2}$, and $p_{3}$ is

$$
\rho_{P}=\left(\begin{array}{ccc}
1 & -0.2 & 0.3 \\
-0.2 & 1 & 0.2 \\
0.3 & 0.2 & 1
\end{array}\right)
$$

The optimization results of four-high rolling mill stand are shown in Table 5. CASE 1 is reliability-based MDO optimization results without considering correlations between uncertainties; CASE 2 is reliability-based MDO optimization results with correlations between uncertainties.

Note from Table 5 that, compared with optimization results obtained by CASE 1 , the objective function increases $5.11 \%$ by using our proposed method. This is consistent with the observation from the mathematical example that the conventional reliability-based MDO method tends to give 


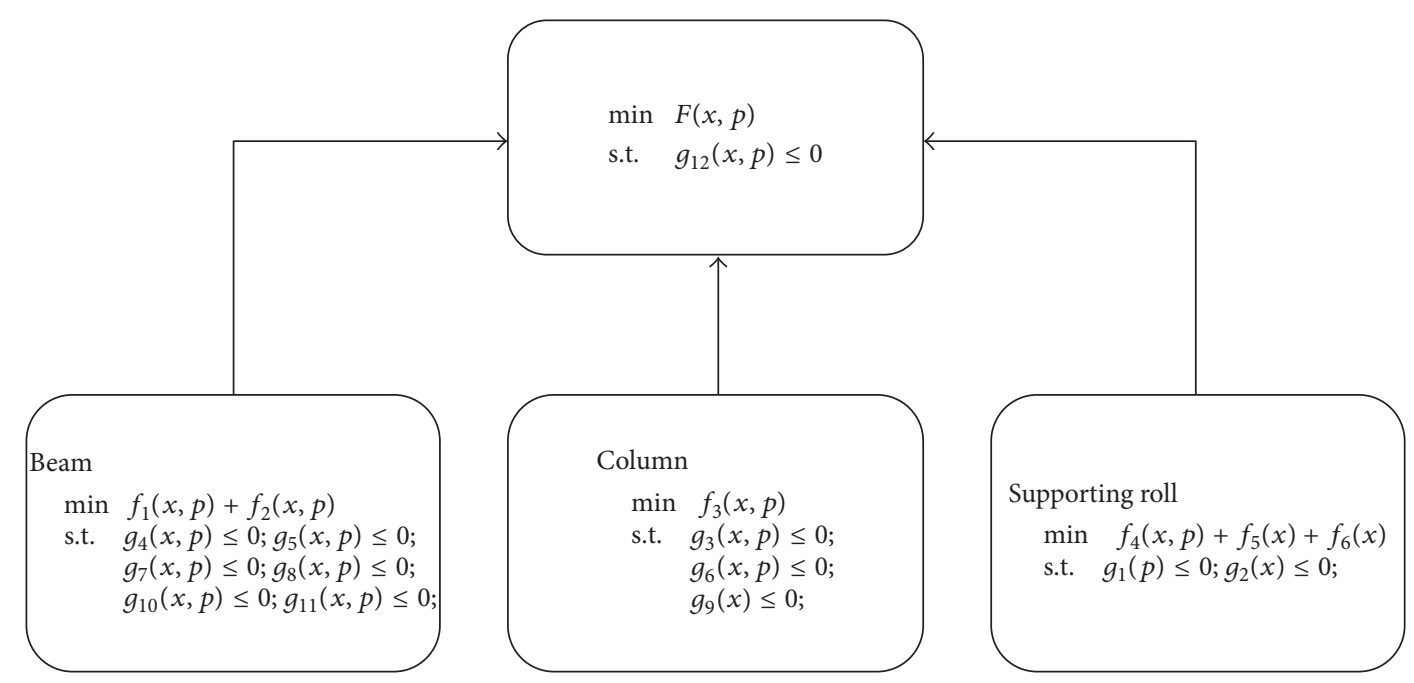

FIGURE 11: The MDO framework of four-high mill stand.

TABle 5: Optimization results.

\begin{tabular}{lccccccccc}
\hline & $x_{1}$ & $x_{2}$ & $x_{3}$ & $x_{4}$ & $f$ & $\operatorname{Pr}_{3}$ & $\operatorname{Pr}_{4}$ & $\operatorname{Pr}_{5}$ & $\operatorname{Pr}_{12}$ \\
\hline CASE 1 & 305 & 665 & 461 & 420 & 1.0210 & 0.9999 & 0.9999 & 0.9999 & 0.9812 \\
CASE 2 & 336 & 653 & 500 & 394 & 1.0732 & 0.9999 & 0.9999 & 0.9999 & 0.9801 \\
\hline
\end{tabular}

inaccurate and optimistic results. Moreover, the proposed method generates results that are more informative and applicable to the engineering reality.

\section{Conclusions}

In this paper through analyzing the characteristics of correlated uncertainties, a quantitative model of correlated uncertainties is established using the ellipsoidal model. The reliability analysis method under correlated uncertainties is investigated and discussed. In addition, a new RBMDO method under correlated uncertainties is proposed. Both a mathematical example and an engineering example are introduced to validate the proposed method.

\section{Conflicts of Interest}

The authors declare that they have no conflicts of interest.

\section{Acknowledgments}

This research is supported by the National Natural Science Foundation of China under the Contract no. 51475082.

\section{References}

[1] X. Du and W. Chen, "An integrated methodology for uncertainty propagation and management in simulation-based systems design," AIAA Journal, vol. 38, no. 8, pp. 1471-1478, 2000.

[2] X. Du and W. Chen, "Efficient uncertainty analysis methods for multidisciplinary robust design," AIAA Journal, vol. 40, no. 3 , pp. 545-552, 2002.
[3] X. Gu, J. E. Renaud, S. M. Batill, R. M. Brach, and A. S. Budhiraja, "Worst case propagated uncertainty of multidisciplinary systems in robust design optimization," Structural and Multidisciplinary Optimization, vol. 20, no. 3, pp. 190-213, 2000.

[4] X. Gu and J. E. Renaud, "Implementation study of implicit uncertainty propagation (IUP) in decomposition-based optimization," in Proceedings of the 9th AIAA/ISSMO Symposium on Multidisciplinary Analysis and Optimization 2002, Atlanta, Ga, USA, September 2002.

[5] S. Sankararaman and S. Mahadevan, "Likelihood-based approach to multidisciplinary analysis under uncertainty," Journal of Mechanical Design, vol. 134, no. 3, Article ID 031008, 2012.

[6] A. Chiralaksanakul and S. Mahadevan, "Decoupled approach to multidisciplinary design optimization under uncertainty," Optimization and Engineering, vol. 8, no. 1, pp. 21-42, 2007.

[7] W. Yao, X. Chen, Q. Ouyang, and M. van Tooren, "A reliabilitybased multidisciplinary design optimization procedure based on combined probability and evidence theory," Structural and Multidisciplinary Optimization, vol. 48, no. 2, pp. 339-354, 2013.

[8] Z. Jiang, W. Li, D. W. Apley, and W. Chen, "A spatial-randomprocess based multidisciplinary system uncertainty propagation approach with model uncertainty," Journal of Mechanical Design, vol. 137, no. 10, Article ID 101402, 2015.

[9] W. Yao, X. Q. Chen, W. C. Luo, M. van Tooren, and J. Guo, "Review of uncertainty-based multidisciplinary design optimization methods for aerospace vehicles," Progress in Aerospace Sciences, vol. 47, no. 6, pp. 450-479, 2011.

[10] G. J. Klir and T. A. Folger, Fuzzy Sets, Uncertainty, and Information, Prentice-Hall, Upper Saddle River, NJ, USA, 1988.

[11] J. C. Helton, "Uncertainty and sensitivity analysis in the presence of stochastic and subjective uncertainty," Journal of Statistical Computation and Simulation, vol. 57, no. 1-4, pp. 3-76, 1997. 
[12] X. Du, "Inverse simulation under uncertainty by optimization," Journal of Computing and Information Science in Engineering, vol. 13, no. 2, Article ID 021005, 2013.

[13] A. Der Kiureghian and O. Ditlevsen, "Aleatory or epistemic? Does it matter?" Structural Safety, vol. 31, no. 2, pp. 105-112, 2009.

[14] H. Agarwal, Reliability based design optimization: formulations and methodologies, University of Notre Dame, Notre Dame, Ind, USA, 2004, Agarwal H. Reliability based design optimization: formulations and methodologies [M]. 2004.

[15] H. Agarwal, J. E. Renaud, E. L. Preston, and D. Padmanabhan, "Uncertainty quantification using evidence theory in multidisciplinary design optimization," Reliability Engineering \& System Safety, vol. 85, no. 1-3, pp. 281-294, 2004.

[16] X. Du and W. Chen, "Concurrent subsystem uncertainty analysis in multidisciplinary design," in Proceedings of the 8th Symposium on Multidisciplinary Analysis and Optimization 2000, Long Beach, Calif, USA, September 2000.

[17] H.-U. Park, J.-W. Lee, J. Chung, and K. Behdinan, "Uncertaintybased MDO for aircraft conceptual design," Aircraft Engineering and Aerospace Technology, vol. 87, no. 4, pp. 345-356, 2015.

[18] H.-U. Park, J. Chung, K. Behdinan, and J.-W. Lee, "Multidisciplinary wing design optimization considering global sensitivity and uncertainty of approximation models," Journal of Mechanical Science and Technology, vol. 28, no. 6, pp. 2231-2242, 2014.

[19] P. T. Lin and H. C. Gea, "Reliability-based multidisciplinary design optimization using probabilistic gradient-based transformation method," Journal of Mechanical Design, vol. 135, no. 2, Article ID 21001, 2013.

[20] J. Ahn and J. H. Kwon, "An efficient strategy for reliability-based multidisciplinary design optimization using BLISS," Structural and Multidisciplinary Optimization, vol. 31, no. 5, pp. 363-372, 2006.

[21] S.-P. Zhu, Y.-J. Yang, H.-Z. Huang, Z. Lv, and H.-K. Wang, "A unified criterion for fatigue-creep life prediction of high temperature components," Proceedings of the Institution of Mechanical Engineers, Part G: Journal of Aerospace Engineering, vol. 231, no. 4, pp. 677-688, 2017.

[22] S. P. Zhu, Q. Lei, and Q. Y. Wang, "Mean stress and ratcheting corrections in fatigue life prediction of metals," Fatigue \& Fracture of Engineering Materials \& Structures, vol. 40, no. 9, pp. 1343-1354, 2017.

[23] Z. Y. Yu, S. P. Zhu, Q. Liu et al., "A new energy-critical plane damage parameter for multiaxial fatigue life prediction of turbine blades," Materials, vol. 10, no. 5, article 513, 2017.

[24] Y. Noh, K. K. Choi, and L. Du, "Reliability-based design optimization of problems with correlated input variables using a Gaussian Copula," Structural and Multidisciplinary Optimization, vol. 38, no. 1, pp. 1-16, 2009.

[25] S. Song, Z. Lu, and Z. Song, "Reliability sensitivity analysis involving correlated random variables by directional sampling," in Proceedings of the International Conference on Quality, Reliability, Risk, Maintenance, and Safety Engineering (ICQR2MSE '11), pp. 845-850, Xi'an, China, June 2011.

[26] S. Song and Z. Lu, "Reliability sensitivity analysis based on directional simulation for systems of multiple design points/ failure modes with normal correlated variables," Acta Aeronautica et Astronautica Sinica, vol. 31, no. 1, pp. 109-118, 2010.

[27] C. Jiang, X. Han, G. Y. Lu, J. Liu, Z. Zhang, and Y. C. Bai, "Correlation analysis of non-probabilistic convex model and corresponding structural reliability technique," Computer Methods
Applied Mechanics and Engineering, vol. 200, no. 33-36, pp. 2528-2546, 2011.

[28] X. Du, "Unified uncertainty analysis by the first order reliability method," Journal of Mechanical Design, vol. 130, no. 9, Article ID 091401, 10 pages, 2008.

[29] D.-B. Meng, H. Xu, X. Zhang, B. Zheng, and H.-Z. Huang, "First-Order Second-Moment based multidisciplinary design optimization," in Proceedings of the International Conference on Quality, Reliability, Risk, Maintenance, and Safety Engineering (ICQR2MSE '11), pp. 920-924, Xi'an, China, June 2011.

[30] O. Ditlevsen and O. Madsen H, Structural Reliability Methods, Wiley, New York, NY, USA, 1996.

[31] A. Der Kiureghian and P.-L. Liu, "Structural reliability under incomplete probability information," Journal of Engineering Mechanics, vol. 112, no. 1, pp. 85-104, 1986.

[32] P.-L. Liu and A. der Kiureghian, "Multivariate distribution models with prescribed marginals and covariances," Probabilistic Engineering Mechanics, vol. 1, no. 2, pp. 105-112, 1986.

[33] X. Qu and R. T. Haftka, "Reliability-based design optimization using probabilistic sufficiency factor," Structural and Multidisciplinary Optimization, vol. 27, no. 5, pp. 314-325, 2004.

[34] D. Meng, Y.-F. Li, H.-Z. Huang, Z. Wang, and Y. Liu, "Reliability-based multidisciplinary design optimization using subset simulation analysis and its application in the hydraulic transmission mechanism design," Journal of Mechanical Design, vol. 137, no. 5, Article ID 051402, 2015.

[35] X. Du, J. Guo, and H. Beeram, "Sequential optimization and reliability assessment for multidisciplinary systems design," Structural and Multidisciplinary Optimization, vol. 35, no. 2, pp. 117-130, 2008.

[36] P. Meng, Y. Li, Z. Jiang, W. Yin, and J. Li, "Structure optimal design of four-high rolling mill stand based on improved collaborative optimization algorithm," International Journal of Advancements in Computing Technology, vol. 5, no. 8, pp. 843851, 2013. 


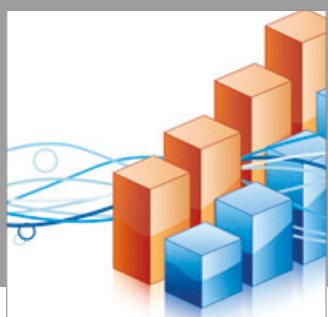

Advances in

Operations Research

vatersals

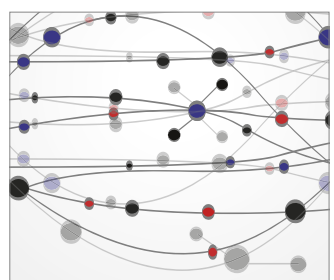

\section{The Scientific} World Journal
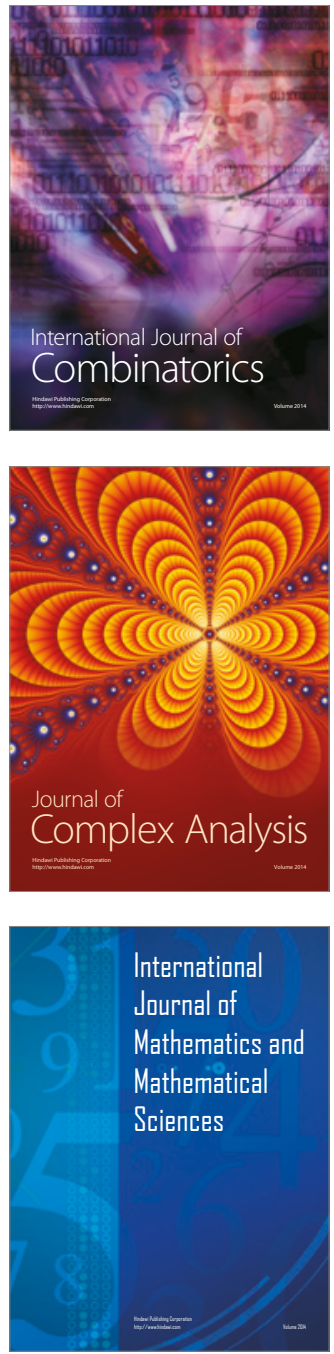
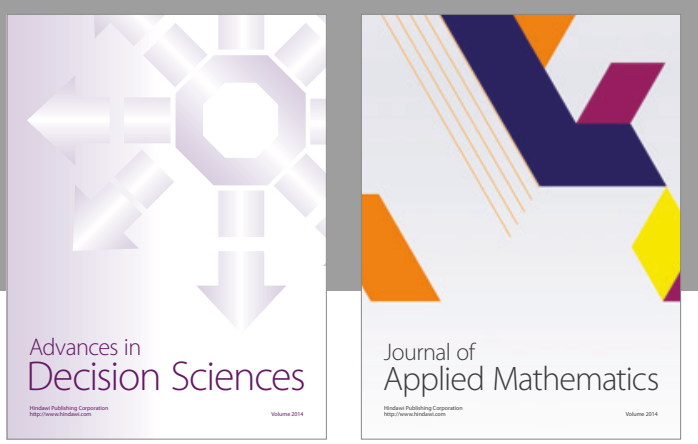

Algebra



\section{Hindawi}

Submit your manuscripts at

https://www.hindawi.com
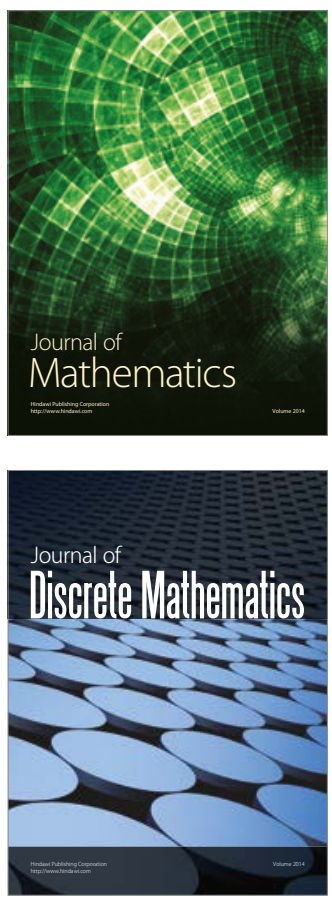

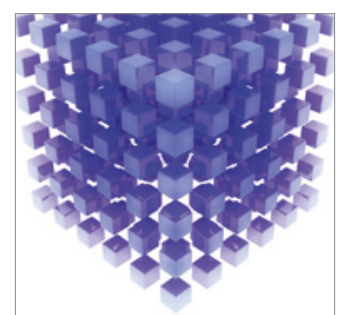

Mathematical Problems in Engineering
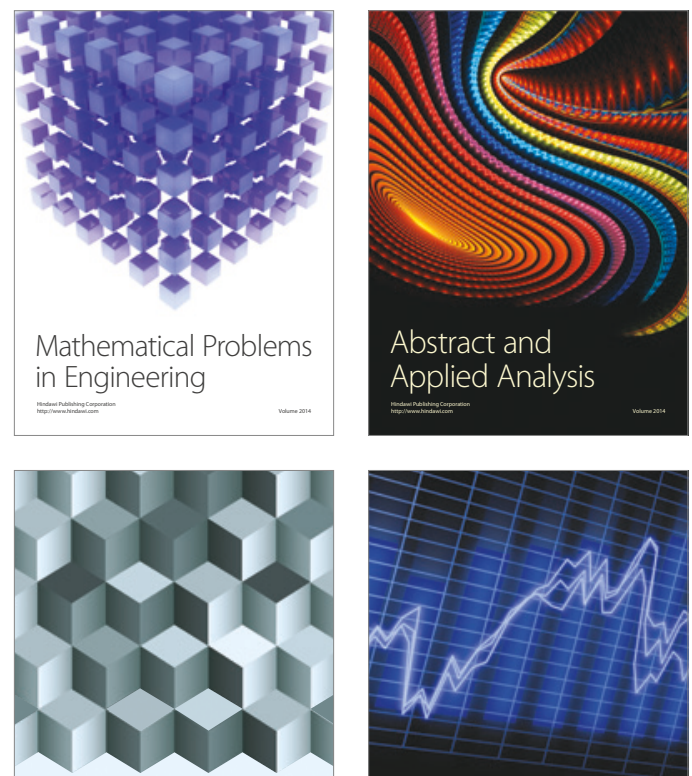

Journal of

Function Spaces

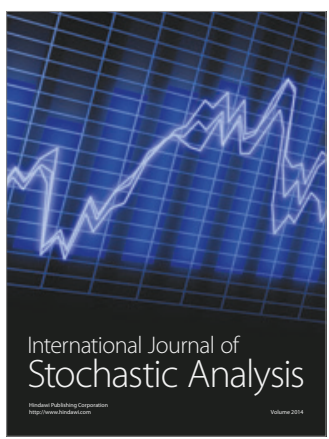

Probability and Statistics
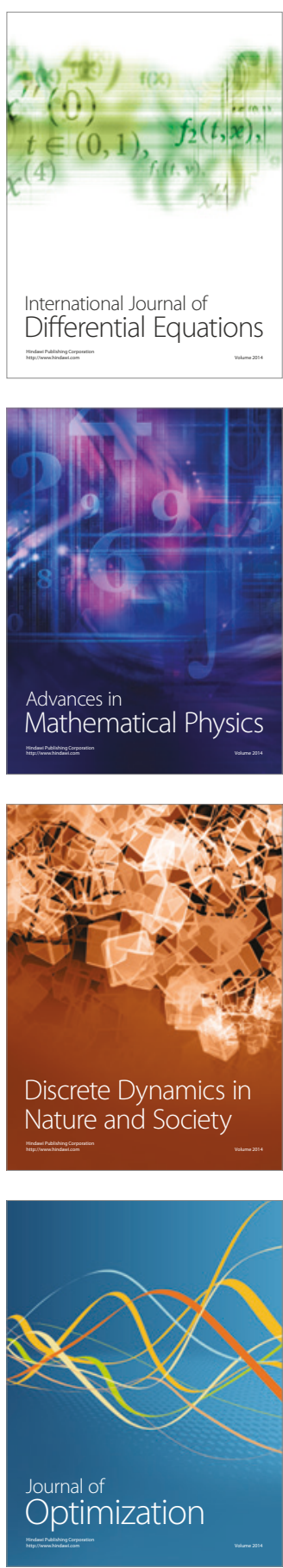